\title{
The prognostic role of hepatic venous pressure gradient in cirrhotic patients undergoing elective extrahepatic surgery
}

\section{Graphical abstract}

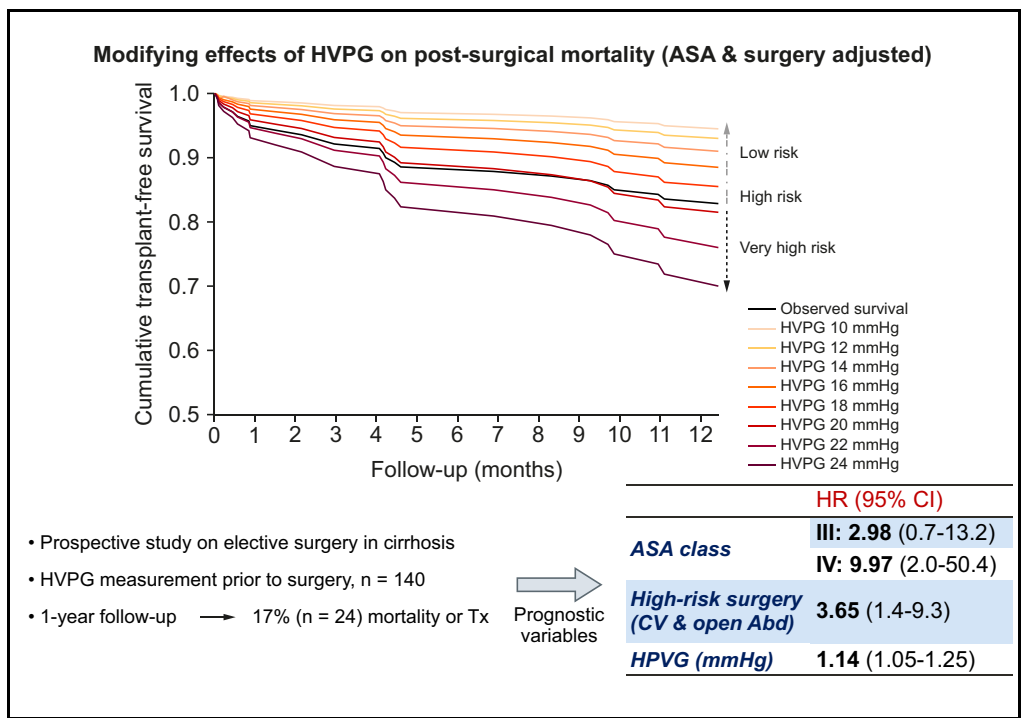

\section{Highlights}

- Hepatic venous pressure gradient is a prognostic factor in cirrhotic patients undergoing surgery.

- ASA class and the type of surgery are the other main prognostic factors.

- Hepatic venous pressure gradient values $>16 \mathrm{mmHg}$ are independently associated with higher mortality.

- Hepatic venous pressure gradient values $\geq 20 \mathrm{mmHg}$ identify the patients at highest risk.

- The potential of pre-surgery TIPS in high-risk patients deserves further study.

\section{Authors}

Enric Reverter, Isabel Cirera, Agustín Albillos, ..., Virginia Hernández-Gea, Jaume Bosch, Joan Carles García-Pagán

\section{Correspondence}

jcgarcia@clinic.cat

(J.C. García-Pagán)

\section{Lay summary}

The hepatic venous pressure gradient is associated with outcomes in patients with cirrhosis undergoing elective extrahepatic surgery. It enables a better stratification of risk in these patients and provides the foundations for potential interventions to improve post-surgical outcomes. 


\title{
The prognostic role of hepatic venous pressure gradient in cirrhotic patients undergoing elective extrahepatic surgery
}

\author{
Enric Reverter ${ }^{1,6}$, Isabel Cirera ${ }^{2}$, Agustín Albillos ${ }^{3,6}$, Wilma Debernardi-Venon ${ }^{4}$, Juan G. Abraldes ${ }^{1,6}$, \\ Elba Llop ${ }^{1,6}$, Alexandra Flores ${ }^{1,6}$, Graciela Martínez-Palli ${ }^{5}$, Annabel Blasi ${ }^{5}$, Javier Martínez ${ }^{3,6}$, \\ Fanny Turon ${ }^{1,6}$, Juan Carlos García-Valdecasas ${ }^{8}$, Annalisa Berzigotti $^{1,6}$, Antoni M. de Lacy ${ }^{7}$, \\ Josep Fuster ${ }^{8}$, Virginia Hernández-Gea ${ }^{1,6}$, Jaume Bosch ${ }^{1,6}$, Joan Carles García-Pagán ${ }^{1,6, *}$ \\ ${ }^{1}$ Barcelona Hepatic Hemodynamic Laboratory, Liver Unit, Hospital Clínic, Institut d'Investigacions Biomèdiques August Pi i Sunyer \\ (IDIBAPS), University of Barcelona, Spain; ${ }^{2}$ Gastroenterology and Hepatology, Hospital del Mar, Barcelona, Spain; ${ }^{3}$ Gastroenterology \\ and Hepatology, Hospital Universitario Ramón y Cajal, Instituto Ramón y Cajal de Investigación Sanitaria (IRYCIS), University of Alcalá, \\ Madrid, Spain; ${ }^{4}$ Department of Gastroenterology, Molinette Hospital, Torino, Italy; ${ }^{5}$ Anesthesiology Department, Hospital Clínic, \\ IDIBAPS, University of Barcelona, Spain; ${ }^{6}$ Centro de Investigaciones Biomédicas en Red de Enfermedades Hepáticas y Digestivas (CIBERehd), \\ Spain; ${ }^{7}$ Gastrointestinal Surgery Department, Hospital Clinic, IDIBAPS, University of Barcelona, Spain; ${ }^{8}$ Hepatobiliary and Pancreatic
} Surgery Department, Hospital Clínic. IDIBAPS, University of Barcelona, Spain

Background \& Aims: Surgery in cirrhosis is associated with a high morbidity and mortality. Retrospectively reported prognostic factors include emergency procedures, liver function (MELD/Child-Pugh scores) and portal hypertension (assessed by indirect markers). This study assessed the prognostic role of hepatic venous pressure gradient (HVPG) and other variables in elective extrahepatic surgery in patients with cirrhosis.

Methods: A total of 140 patients with cirrhosis (Child-Pugh A/B/ C: $59 / 37 / 4 \%)$, who were due to have elective extrahepatic surgery (121 abdominal; 9 cardiovascular/thoracic; 10 orthopedic and others), were prospectively included in 4 centers (20022011). Hepatic and systemic hemodynamics (HVPG, indocyanine green clearance, pulmonary artery catheterization) were assessed prior to surgery, and clinical and laboratory data were collected. Patients were followed-up for 1 year and mortality, transplantation, morbidity and post-surgical decompensation were studied.

Results: Ninety-day and 1-year mortality rates were $8 \%$ and $17 \%$, respectively. Variables independently associated with 1-year mortality were ASA class (American Society of Anesthesiologists), high-risk surgery (defined as open abdominal and cardiovascular/thoracic) and HVPG. These variables closely predicted 90-, 180- and 365-day mortality (C-statistic >0.8). HVPG values $>16 \mathrm{mmHg}$ were independently associated with mortality and values $\geq 20 \mathrm{mmHg}$ identified a subgroup at very high risk of death (44\%). Twenty-four patients presented persistent or de novo decompensation at 3 months. Low body mass index, Child-Pugh class and high-risk surgery were associated

Keywords: Portal hypertension; Surgical risk; Post-operative complications; HVPG; Prognosis; Modeling.

Received 9 April 2019; received in revised form 4 July 2019; accepted 6 July 2019

* Corresponding author. Address: Hepatic Hemodynamic Laboratory, Liver Unit, Hospital Clínic, Villarroel 170, 08036 Barcelona, Spain. Tel.: +34 932275400x2209, fax: +34934515272.

E-mail address: jcgarcia@clinic.cat (J.C. García-Pagán). with death or decompensation. No patient with HVPG $<10 \mathrm{mmHg}$ or indocyanine green clearance $>0.63$ developed decompensation.

Conclusions: ASA class, HVPG and high-risk surgery were prognostic factors of 1-year mortality in cirrhotic patients undergoing elective extrahepatic surgery. HVPG values $>16 \mathrm{mmHg}$, especially $\geq 20 \mathrm{mmHg}$, were associated with a high risk of post-surgical mortality.

Lay summary: The hepatic venous pressure gradient is associated with outcomes in patients with cirrhosis undergoing elective extrahepatic surgery. It enables a better stratification of risk in these patients and provides the foundations for potential interventions to improve post-surgical outcomes.

(C) 2019 European Association for the Study of the Liver. Published by Elsevier B.V. All rights reserved.

\section{Introduction}

Cirrhosis is a life-threatening condition and a major cause of morbidity and mortality worldwide. Improvements in the management of its related complications, of its etiologies (i.e. viral eradication), and the option of liver transplantation have increased life expectancy of patients with cirrhosis. In this setting, it is not unusual that major surgical procedures are proposed for patients with cirrhosis to address orthopedic, malignancy or cirrhosis related complications. In fact, patients with cirrhosis have a high incidence of gallstones and abdominal wall hernias that require surgical repair. ${ }^{1-4}$ Surgery in cirrhosis has always been associated with high perioperative morbidity (about 30\%, including infections, renal failure, decompensation, blood transfusion, re-intervention, etc.) and mortality, ranging from 10 to $30 \%$ in the most recent series. ${ }^{5-11}$ The main factors associated with these poor outcomes have been related to liver function (Child-Pugh or model for end-stage liver disease [MELD] scores), to the type of surgery (higher risk in open abdominal, cardiovascular and thoracic surgeries), and to the presence of signs or symptoms of portal hypertension (PHT) ${ }^{5,8,10-14}$ However, there are no universally accepted 
prospective scores to assess surgical risk for patients with cirrhosis. The most widely accepted score is probably that from the Mayo Clinic, based on MELD, ASA class and age. ${ }^{11,15}$ Although it was developed in a very large cohort, this model combines emergency and elective surgery, combining different profiles of patients that may act as confounding factors (for example, MELD score is usually higher in emergency surgery patients). The major weakness of prognostic studies of surgery in cirrhosis is their retrospective nature and the lack of prospective validation studies.

Development of PHT in cirrhosis is associated with marked systemic and splanchnic hemodynamic disturbances that progress in parallel to cirrhosis and are of prognostic significance. ${ }^{16}$ These disturbances impact on cardiopulmonary and renal circulation and may contribute to post-surgical complications. Although PHT has been evaluated in several studies, it has always been done by means of indirect signs (clinical, laboratory or imaging) such as the presence of splenomegaly, ascites, encephalopathy, esophageal varices or a low platelet count. ${ }^{9,10}$ Although the presence of these signs in cirrhosis is unequivocally associated with clinically significant PHT, it may also be present in their absence. ${ }^{17,18}$ In addition, studies assessing the prognostic value of PHT in the natural history of cirrhosis have identified different risk thresholds. Indeed, most clinical events occurring in cirrhosis are associated with the degree of PHT: ascites and collateral formation for HVPG values $\geq 10 \mathrm{mmHg}$, variceal bleeding when $\geq 12 \mathrm{mmHg}$, and worse prognosis if $\geq 20 \mathrm{mmHg}$ for variceal bleeding. ${ }^{18}$ HVPG has an important value for prognostic stratification in surgery for hepatocellular carcinoma (HCC), but studies assessing its prognostic value in extrahepatic surgery are lacking. ${ }^{18,19}$ In this regard, accurate assessments of the severity of PHT and of liver function by HVPG measurement and indocyanine green clearance, respectively, might reveal more sensitive prognostic factors for postsurgical morbidity and mortality in cirrhosis. ${ }^{19,20}$

The aim of the present study was to define the prognostic role of HVPG and other variables in a prospective cohort of patients with cirrhosis undergoing elective extrahepatic surgery.

\section{Patients and methods}

The present study is a prospective multicenter cohort study assessing the prognostic role of HVPG and of other variables in extrahepatic surgery in cirrhosis. The study was conducted in 4 university hospitals (Hospital Clinic-Barcelona, Hospital del Mar-Barcelona, Hospital Ramón y Cajal-Madrid, and Hospital Molinette-Torino) with expertise in the field of cirrhosis and in surgical procedures in these patients. Inclusion criteria were: liver cirrhosis of any etiology; elective extrahepatic surgery planned within 3 months and requiring regional or general anesthesia; informed consent of the patient. The exclusion criteria were as follows: emergent surgery; liver resection and portal hypertension surgery; terminal hepatic or extrahepatic disease with expected survival lower than 6 months. The study was approved by the Ethics Committee for Clinical Investigation of the Hospital Clinic (registry number 6/3/2002) as well as by each participating hospital Ethics Committee's. The study was conducted following the principles of the Declaration of Helsinki.

One hundred forty patients with cirrhosis planned for elective extrahepatic surgery were prospectively included between
July 2002 and June 2011. Baseline clinical, hemodynamic and laboratory data were collected, and patients were followed-up for 1 year after surgery, or until death/transplantation, whichever occurred first. Within the 3 months prior to surgery, patients underwent the HVPG measurement. During hospitalization for surgery, patients were closely monitored to register clinical course, development of post-surgical complications and mortality. Complications developed during hospitalization, length of stay, blood product transfusion and supportive therapies (mechanical ventilation, renal replacement therapy, etc.) were registered. After discharge, patients were visited at 6 weeks, 3 months and every 3 months up to 1 year, or whenever needed. Clinical and laboratory variables were collected at month 6 and 12 after surgery.

\section{Hemodynamic studies}

Within the 3 months prior to surgery, patients underwent a hemodynamic study, which included the assessment of hepatic and cardiopulmonary pressures and hepatic blood flow by infusion of indocyanine green. The studies were performed in fasting conditions, under local anesthesia with anxiolytic doses of midazolam (0.01-0.02 mg/kg). All patients underwent standard non-invasive monitoring by continuous display of heart rate, pulse oximetry, and respiratory rate along with blood pressure (5 min). The right jugular vein was canalized under ultrasonographic guidance (SonoSite Inc, Bothell, WA) with an 8F catheter introducer (Axcess; Maxxim Medical, Athens, TX) by Seldinger technique. Initially, under fluoroscopic control a pulmonary artery catheter (Edwards Lifesciences, LLC, CA) was used to measure cardiopulmonary pressures and cardiac output. After that, HVPG measurement was performed as previously described. ${ }^{21}$ In brief, a 7F balloon-tipped catheter ("Fogarty" Edwards Lifesciences LLC, CA) was guided into the main right or middle hepatic vein for measurements of wedged (occluded) (WHVP) and free hepatic venous pressures (FHVP). The HVPG results from the difference between WHVP and FHVP. The adequacy of occlusion was checked by gentle injection of a small amount of radiologic contrast medium after balloon inflation. All measurements were taken by triplicate and averaged to obtain the baseline HVPG. Permanent tracings were obtained in each case in a multichannel recorder (GE Healthcare, Milwaukee, WI).

Hepatic blood flow was measured by the Fick principle during a continuous infusion of indocyanine green, as previously described. $^{22}$ Briefly, preceded by a priming dose (5 mg), a solution of indocyanine green (Pulsion Medical Systems, Munich, GE) was infused intravenously at a constant rate of $0.2 \mathrm{mg} / \mathrm{min}$. After an equilibration period of at least $40 \mathrm{~min}, 4$ separate sets of simultaneous $3 \mathrm{ml}$ samples of peripheral and hepatic venous blood were obtained for the measurement of hepatic blood flow (HBF). Hepatic clearance of indocyanine green, fractional clearance, and hepatic intrinsic clearance were used as quantitative liver function tests.

Plasma renin activity was also studied as an index of effective hypovolemia. Systemic and pulmonary vascular resistance indexes (dyn.s $/ \mathrm{cm}^{5} \cdot \mathrm{m}^{2}$ ) were calculated as follows, respectively: (mean arterial pressure - right atrial pressure $[\mathrm{mmHg}]) \cdot 79.9 /$ cardiac index $\left[\mathrm{L} \cdot \mathrm{min}^{-1} \cdot \mathrm{m}^{-2}\right]$ and (pulmonary artery pressure - pulmonary capillary wedge pressure $[\mathrm{mmHg}]) \cdot 79.9 /$ cardiac index $\left[\mathrm{L} \cdot \mathrm{min}^{-1} \cdot \mathrm{m}^{-2}\right]$, respectively. Hepatic sinusoidal resistance (HSR) was estimated as $\mathrm{HSR}=\mathrm{HVPG} \times 79.9 / \mathrm{HBF}$. 


\section{Outcome measures}

The primary endpoint of the study was to evaluate postoperative mortality at 90 days and up to 1 year of follow-up. Secondary endpoints were the development of post-surgical complications during hospitalization and de novo or worsening hepatic decompensation lasting beyond 3 months after surgery. The following events were considered main post-surgical complications: new or worsening acute kidney injury (increase of creatinine $>50 \%$ or above $1.5 \mathrm{mg} / \mathrm{dl}$ ), post-surgery hemodynamic instability (any hypotension in the first $24 \mathrm{~h}$ requiring vasoactive drugs and/or volume expansion), post-surgical bleeding requiring transfusion, hepatic encephalopathy, infections (urinary, wound, respiratory, catheter, etc.), re-intervention, intubation longer than $24 \mathrm{~h}$, development of distress respiratory syndrome, re-intubation, deep vein thrombosis or pulmonary embolism. Intensive care unit and hospital length of stay were registered.

In previously compensated patients, de novo decompensation was defined as jaundice or ascites persisting beyond 90 days from surgery, as well as any post-surgical encephalopathy, PHT-related bleeding or spontaneous bacterial peritonitis. Mild ascites through drainages not requiring diuretics and not persisting at day 90 was not considered. In previously decompensated patients, decompensation was defined as any new type of decompensation or worsening from baseline (i.e. increased number of episodes/intensity of hepatic encephalopathy, need for paracentesis or increased diuretic dose in patients with previous ascites, new PHT-related bleeding).

The study of 90-day and 1-year transplant-free survival (endpoint mortality or transplantation) was done by multivariable survival Cox regression analysis. To assess the performance and accuracy of predictions, including the significant variables, Harrell C-statistic (3, 6 and 12 months) and Akaike information criterion (AIC) were calculated. Harrell C-statistic is a natural extension of the AUROC curve in the context of censored data in survival analysis with binary endpoints. AIC estimates the relative quality of statistical models for a given data set based on likelihoods, the preferred model being the one with the lowest AIC value. Two sub-analyses were performed: i) in the abdominal subgroup, which was predominant in our cohort (121/140 patients); and ii) in the high-risk surgery group, which accounted for the majority of events.

A competing-risk regression analysis (Fine and Gray method) was performed to control for the potential bias of liver transplantation. This approach was also used to further understand and delineate the contribution of each prognostic variable according to the specific cause of death at follow-up: liver or non-liver-related death.

The analysis of persistent/de novo decompensation was performed using the Student's $t$ test for parametric continuous variables and the Wilcoxon rank sum test for non-parametric continuous variables. Categorical variables were assessed by the Chi-square test or the Fisher's exact test where appropriate. Three-month decompensation was analyzed both including or not including mortality (considering death as the worst decompensation). Multivariable analysis was performed by logistic regression including variables with a $p$ value $<0.05$ at univariate analysis.

To optimize multivariable analysis, based on our cohort size and on previous studies of surgical risk in cirrhosis, surgery types were grouped into high-risk and low-risk procedures. The high-risk group comprised cardiovascular, thoracic and open abdominal surgeries, while the low-risk group comprised laparoscopic and abdominal wall surgeries, orthopedic and others. Child-Pugh and MELD scores were not simultaneously included at multivariable analysis due to their collinearity.

Quantitative variables were expressed as mean \pm standard deviation and categorical variables as $\mathrm{n}(\%)$. Statistical significance was established at a 2-tailed $p$ value of less than 0.05 . Analysis was performed with IBM SPSS Statistics 20.0 package (SPSS Inc., Chicago, IL); survival nomogram was built with $\mathrm{R}$ (version 3.5.1) and competing-risk analysis was performed with the command "UAB Competing Risks" developed by the Applied Biostatistics Laboratory (Autonomous University of Barcelona).

\section{Results}

\section{Study cohort, procedures and outcomes}

Between July 2002 and June 2011, 140 patients undergoing elective extrahepatic surgery were prospectively included (Fig. 1). Table 1 shows patients' baseline characteristics and Table 2 details surgical information and short and long-term outcomes. Two patients with transjugular intrahepatic portosystemic shunt (TIPS) and 1 patient with surgical shunt were included (bleeding indication years before surgery, portal gradient between 7.5-10 mmHg, 2 survived and 1 died at follow-up). Median follow-up after surgery was 360 days and mortality or transplantation at 90 days was $8 \%$ (11/140 patients) and $17 \%$ at 1 year (24/140 patients: 21 deaths and 3 orthotopic liver transplants [OLTs]).

\section{0-day and 1-year follow-up mortality/OLT}

Eleven patients died during the 90 days after surgery. ASA class and high-risk surgery were significantly associated with 90-day mortality while HVPG and right atrial pressure showed a trend to statistical significance ( $p=0.059$, both). No further multivariable analysis was done due to the low number of events.

At 1-year follow-up, 21 patients died and 3 underwent OLT. Variables associated with mortality/OLT were ASA class, highrisk surgery, HVPG, intrinsic indocyanine green clearance, renin activity, albumin, Child-Pugh score and previous decompensa-

July 2002-June 2011:

165 patients with cirrhosis planned

for elective extra-hepatic surgery

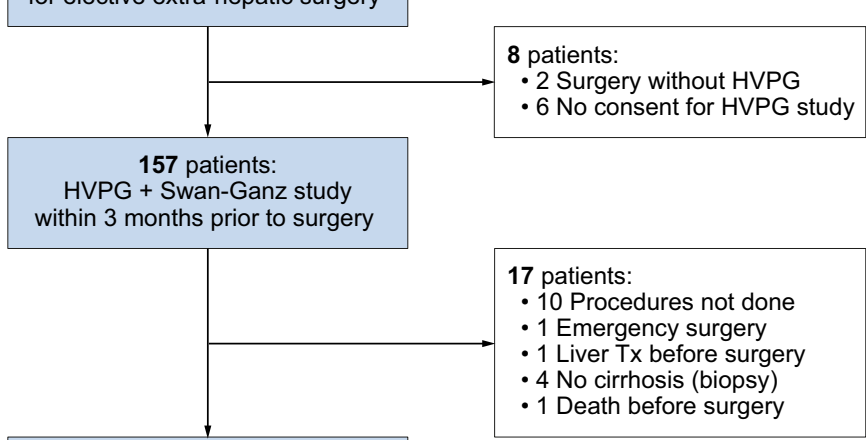

Study cohort:

140 patients with cirrhosis

undergoing elective surgery

Prospective 1 year follow-up

Fig. 1. Flowchart of the patients included in the study. HVPG, hepatic venous pressure gradient. 
Table 1. Baseline clinical and laboratory characteristics of patients in the study $(\mathrm{N}=140)$.

\begin{tabular}{|c|c|}
\hline \multicolumn{2}{|l|}{ Characteristics } \\
\hline Age (years) & $62 \pm 8$ \\
\hline Male gender, $\mathrm{n}(\%)$ & $92(66)$ \\
\hline Etiology alcohol/viral/others, n (\%) & $59(42) / 54(39) / 27(19)$ \\
\hline Previous decompensation, $\mathrm{n}(\%)$ & $84(60)$ \\
\hline Non-selective $\beta$-blockers, $\mathrm{n}(\%)$ & $51(36)$ \\
\hline Diuretics, n (\%) & $63(45)$ \\
\hline Hepatic venous pressure gradient $(\mathrm{mmHg}$ ) & $15.0 \pm 5.4$ \\
\hline HVPG $\geq 10 \mathrm{mmHg}, \mathrm{n}(\%)$ & $116(83)$ \\
\hline Child-Pugh class A/B/C, n (\%) & $83(59) / 51(37) / 6(4)$ \\
\hline TIPS or surgical shunt, $\mathrm{n}(\%)$ & $3(2)$ \\
\hline MELD score (UNOS) & $11.1 \pm 3.2$ \\
\hline MELD-Na score & $12.1 \pm 4.1$ \\
\hline Previous surgeries, n (\%) & $82(59)$ \\
\hline Charlson comorbidity index & $5.0 \pm 1.8$ \\
\hline Malignancy, n (\%) & $63(45)$ \\
\hline ASA class II/III/IV, n (\%) & $43(31) / 85(60) / 12(9)$ \\
\hline Body mass index $\left(\mathrm{kg} / \mathrm{m}^{2}\right)$ & $27.1 \pm 6.2$ \\
\hline Creatinine (mg/dl) & $1.0 \pm 0.3$ \\
\hline $\mathrm{Na}(\mathrm{mEq} / \mathrm{L})$ & $138 \pm 4.1$ \\
\hline Bilirubin (mg/dl) & $1.7 \pm 1.6$ \\
\hline $\operatorname{ALT}(\mathrm{U} / \mathrm{L})$ & $51 \pm 57$ \\
\hline AST (U/L) & $61 \pm 48$ \\
\hline Albumin $(\mathrm{g} / \mathrm{L})$ & $36.3 \pm 6.4$ \\
\hline Hemoglobin (g/L) & $118 \pm 28$ \\
\hline Leucocyte $\left(\mathrm{x} 10^{9} / \mathrm{L}\right)$ & $5.4 \pm 2.4$ \\
\hline Platelets $\left(\mathrm{x} 10^{12} / \mathrm{L}\right)$ & $119 \pm 67$ \\
\hline Prothrombin activity (\%) & $73 \pm 15$ \\
\hline INR & $1.22 \pm 0.23$ \\
\hline
\end{tabular}

Results presented as mean \pm SD unless otherwise indicated.

ALT, alanine aminotransferase; ASA, American Society of Anesthesiologists; AST, aspartate aminotransferase; HVPG, hepatic venous pressure gradient; INR, international normalized ratio; MELD, model for end-stage liver disease; TIPS, transjugular intrahepatic portosystemic shunt; UNOS, United Network for Organ Sharing.

tion. At multivariable analysis ASA class (hazard ratio [HR] III vs. $\mathrm{II}=2.98 ;$ HR IV vs. II =9.97; $p=0.008)$, high-risk surgery $(\mathrm{HR}=3.65 ; p=0.006)$ and HVPG $(\mathrm{HR}=1.14 ; p=0.003)$ remained as independent prognostic factors (Table 3 ). When assessing the accuracy of potential predictions by these variables, Harrell C-statistic was very satisfactory for predictions at days 90, 180 and 365 (>0.85 for all, Table 4 ).

A predictive nomogram for post-surgical mortality was created with these 3 variables (Fig. 2). Probabilities of death at time " $t$ " can be also estimated by the equation $\mathrm{P}=1-\mathrm{e}^{(-\mathrm{CBH}(\mathrm{t}) \times \exp (\beta(\mathrm{t})) \times \text { RiskPoints) }}$. Risk Points for each patient are calculated from Cox regression coefficients for prognostic variables as follows: $0.013 \times \mathrm{HVPG}+$ ASA class points $(\mathrm{II}=0$, $\mathrm{III}=1.092, \mathrm{IV}=2.3)+$ risky surgery points $(\mathrm{No}=0$, Yes $=1.296)$. $\mathrm{CBH}(\mathrm{t})$ and $\beta(\mathrm{t})$ are the cumulated baseline hazard and the score coefficient estimated by the model fitted for time $t$. At the time points 180 and 365 days, these values are: $\mathrm{CBH}(180)=0.00093$, $\beta(180)=1.151 ; \mathrm{CBH}(365)=-0.00508, \beta(365)=1$.

Although only 3 patients reached OLT instead of death, a further competing-risk analysis was performed. Previously identified variables were again independently associated with 1-year mortality: ASA class ( $\mathrm{sHR}=2.73 ; p=0.005)$, high-risk surgery $(\mathrm{sHR}=6.24 ; p<0.001)$ and HVPG $(\mathrm{sHR}=1.13 ; p=0.019)$. Since all 3 patients undergoing OLT were in the low-risk surgery group, sHR for high-risk surgery was higher than that at Cox regression.
Specific mortality analysis: liver and non-liver-related events Among events, 15 deaths were related to liver events, 6 deaths were not related to liver events, and the indication of the 3 patients undergoing OLT was de novo HCC (they were analyzed as non-liver-related events). When previously identified prognostic variables were analyzed for the specific cause of death, HVPG $(\mathrm{sHR}=1.23 ; \quad p=0.001)$ and high-risk surgery ( $\mathrm{sHR}=11.28 ; p<0.001)$ were associated with liver-related events $(n=15)$. When analyzing not liver-related events, ASA class $(n=9)$ was the only associated variable $(\mathrm{sHR}=5.39$; $p<0.001$ ). Table S1 further details this analysis.

\section{Severity of portal hypertension and mortality: high- and low- risk thresholds}

After identifying HVPG as a prognostic factor, we assessed the potential existence of different thresholds of risk for HVPG. Fig. 3 shows the modifying effect of HVPG on expected mortality as adjusted by ASA and type of surgery. HVPG was therefore dichotomized and different cut-offs were studied at Cox regression along with ASA class and type of surgery. A significant increase in mortality was found for HVPG values $>16 \mathrm{mmHg}$ (HR >2.5). HVPG values $\geq 20 \mathrm{mmHg}$ (HR 5.67, $p<0.001$ ) identified an especially high-risk group of patients: 14 of 32 patients above this value died/required OLT during follow-up (Fig. 4). This cut-off ( $\geq 20 \mathrm{mmHg}$ ) was the most efficient when constructing a predictive model with ASA and type of surgery: it showed a similar performance (lowest AIC, equal C-statistic) to that shown by the continuous HVPG model (Table 4).

\section{Abdominal and high-risk surgery sub-analysis}

Among the 121 abdominal surgeries there were 54 laparoscopic (23 colectomies, 20 cholecystectomies, 6 gastrectomies, 2 exploratory, 1 splenectomy, 1 nephrectomy and 1 ileal resection), 41 open abdominal and 26 abdominal wall procedures. Mortality/OLT at 3 months and at 1-year were 7\% (9/121) and $16 \%$ (19/121), respectively. Mortality was low among laparoscopic procedures: 0 events at 90 days, 1 death and 2 OLTs at 1 year. Survival analysis confirmed the previously reported prognostic variables: ASA class (HR III vs. II $=2.44$; IV $v s$. $\mathrm{II}=16.4 ; \quad p=0.002)$, open abdominal surgery $\quad(\mathrm{HR}=4.3$; $p=0.001)$ and HVPG $(\mathrm{HR}=1.18, p=0.001)$. The C-statistic for predictions with these variables was $0.909,0.895$ and 0.840 at days 90, 180 and 365, respectively (Table 4). In this subgroup, an HVPG of $20 \mathrm{mmHg}$ was again the most efficient threshold to detect patients at very high risk. The performance of both prognostic models with a continuous or dichotomous HVPG ( $\geq 20 \mathrm{mmHg}$ ) were equivalent (Table 4).

Among the 50 patients in the high-risk surgery group (open abdominal, cardiovascular/thoracic), 16 died during follow-up. ASA class and HVPG were independent prognostic factors with similar HR to the overall cohort: 3.3 (III vs. II) and 8.8 (IV vs. II) for ASA $(p=0.016)$ and $1.13(p=0.05)$ for HVPG. An HVPG $\geq 20 \mathrm{mmHg}$ was the most efficient cut-off, along with ASA (HR $6.06 ; p=0.001)$. Fig. S1 shows the effects of very high-risk HVPG and ASA class as stratified by type of surgery.

\section{De novo or worsening decompensation at 3 months}

At 3 months after surgery, 32 patients had at least 1 persistent de novo or worsening decompensation. Twenty-two developed ascites, 8 presented with hepatic encephalopathy, 3 spontaneous bacterial peritonitis, 2 variceal bleeding, and 8 of them finally died within the 3 -month period. Three additional 
Table 2. Surgical procedures, outcomes, decompensation, 90-day and 1year follow-up mortality.

\begin{tabular}{|c|c|}
\hline Surgical procedures & \\
\hline Overall surgical time (minutes), median (IQR) & $120(115)$ \\
\hline Open abdominal; $\mathrm{n}=41$ & $216(120)$ \\
\hline Laparoscopic abdominal; $\mathrm{n}=54$ & $105(80)$ \\
\hline Abdominal wall surgery; $n=26$ & $74(63)$ \\
\hline Cardiovascular and thoracic; $\mathrm{n}=9$ & $210(185)$ \\
\hline Orthopedic (arthroplasty/fracture repair); $\mathrm{n}=7$ & $90(50)$ \\
\hline Others; $\mathrm{n}=3$ & $120(-)$ \\
\hline General/locoregional anesthesia, $\mathrm{n}(\%)$ & $\begin{array}{c}127(91) / 13 \\
(9)\end{array}$ \\
\hline ICU admission, $\mathrm{n}(\%)$ & $36(26)$ \\
\hline ICU length-of-stay (days), median (IQR) & $3(4)$ \\
\hline $\begin{array}{l}\text { Patients receiving blood-derived products transfusion, } \mathrm{n} \\
(\%)\end{array}$ & $61(44)$ \\
\hline Packed red blood cells (units), median (IQR); $\mathrm{n}=52$ & $2(3)$ \\
\hline Fresh frozen plasma (ml), median (IQR); $\mathrm{n}=31$ & $1,000(500)$ \\
\hline Platelets (pools), median (IQR); $\mathrm{n}=25$ & $2(3)$ \\
\hline Perioperative morbidity (any), n (\%) & $69(49)$ \\
\hline Perioperative hypotension, n (\%) & $25(18)$ \\
\hline Perioperative bleeding, $\mathrm{n}(\%)$ & $21(15)$ \\
\hline Perioperative respiratory failure, $\mathrm{n}(\%)$ & $10(7)$ \\
\hline Surgical reintervention, $\mathrm{n}(\%)$ & $4(3)$ \\
\hline Arrhythmia, n (\%) & $8(6)$ \\
\hline Acute kidney injury, $\mathrm{n}(\%)$ & $17(12)$ \\
\hline Ionic disturbances, $\mathrm{n}(\%)$ & $20(14)$ \\
\hline Wound infection, $\mathrm{n}(\%)$ & $8(6)$ \\
\hline Post-operative infection, $\mathrm{n}(\%)$ & $27(19)$ \\
\hline $\begin{array}{l}\text { Persistent de novo/worsening 3-month decompensation, } \mathrm{n} \\
\text { (\%) }\end{array}$ & $24(17)$ \\
\hline Ascites, $\mathrm{n}(\%)$ & $22(16)$ \\
\hline Encephalopathy, n (\%) & $8(6)$ \\
\hline Spontaneous bacterial peritonitis, $\mathrm{n}(\%)$ & $3(2)$ \\
\hline Variceal bleeding, n (\%) & $2(1)$ \\
\hline Post-operative hospital length-of-stay (days), median (IQR) & $6(9)$ \\
\hline In-hospital mortality, n (\%) & $7(5)$ \\
\hline 90-day mortality, n (\%) & $11(8)$ \\
\hline 1-year mortality/OLT, n (\%) & $24(18)$ \\
\hline Post-operative follow-up (days), median (IQR) & $360(42)$ \\
\hline
\end{tabular}

ICU, intensive care unit; OLT, orthotopic liver transplant.

patients died without previous decompensation (sudden death, cardiogenic shock and massive hemoperitoneum). Overall, 35 patients reached the combined endpoint (death/decompensation), which was associated with body mass index (BMI), ASA class, high-risk surgery, MELD, international normalized ratio, albumin, Child-Pugh score, indocyanine green fractional clearance and intrinsic clearance, HVPG, and pre-surgical decompensation (Table S2). At multivariable analysis, a lower BMI, ChildPugh score and high-risk surgery were independently associated with 3-month death or decompensation. When entering MELD (or MELD-Na) instead of Child-Pugh score, variables independently associated with decompensation were BMI, high-risk surgery and HVPG $(p=0.018)$.

Four of the $24(17 \%)$ patients alive and decompensated at 3 months died during the posterior follow-up, while 9 of 105 patients (9\%) died in the non-decompensated group $(p=0.235)$. A time-dependent analysis of decompensation adjusted by ASA, high-risk surgery and HVPG found no association with follow-up mortality ( $\mathrm{HR}=1.12$; CI $0.34-3.62$; $p=0.855$ ).

As an event associated with decompensated or complicated cirrhosis, post-surgical acute kidney injury was present in 17 patients, 12 of whom had moderate-tense ascites (Table 2). AKI was associated with other surgical complications and 8 patients died in the first 90 days. At follow-up, only 3 of 9 living patients persisted with renal dysfunction (creatinine values increase by between $55-100 \%$ from baseline), but none required dialysis.

HVPG, intrinsic and fractional indocyanine green clearances were specifically analyzed. All decompensated patients had HVPG values $\geq 10 \mathrm{mmHg}$. However, at multivariable analysis (along with Child-Pugh score, BMI and high-risk surgery), neither this nor other potential cut-offs were independently associated with decompensation. Regarding indocyanine greenderived values (available in 115 patients), an indocyanine green fractional clearance equal or greater than 0.63 identified patients (20/115) with no risk of decompensation (Youden Index of 0.243 ). At multivariable analysis, this cut-off was an independent predictor of decompensation along with ChildPugh score and BMI ( $p$ value $=0.004$ ).

\section{Discussion}

The present prospective study shows for the first time the prognostic impact of HVPG on extrahepatic surgery in cirrhosis. Besides HVPG, other prognostic variables for post-surgical mortality were ASA class and type of surgery (high vs. low risk), which had previously been reported. ${ }^{6,11,23}$ Importantly, our study is, to date, the first to specifically assess the impact of PHT by prospectively performing HVPG measurements prior to surgery. In previous studies, most of them retrospective, PHT was indirectly defined by signs such as a low platelet count, splenomegaly, varices, ascites or previous decompensation. ${ }^{6,10,12,24,25}$ Although these signs are highly predictive of PHT, the contrary is not always true: more than $50 \%$ of compensated patients with HVPG $>10 \mathrm{mmHg}$ may have no varices and normal or almost normal platelet count. ${ }^{26}$ In our study, a detailed assessment of HVPG clearly shows that the severity of PHT plays a major prognostic role in cirrhotic patients undergoing surgery. A further dichotomous characterization of HVPG allowed for the identification of relevant cut-offs, $>16 \mathrm{mmHg}$ and $\geq 20 \mathrm{mmHg}$ : patients within these values were at high and very high risk of death, respectively. Along with HVPG, ASA class and the type of surgery (high risk vs. low risk) were the other prognostic variable repeatedly reported in previous studies. In our cohort, these 3 variables showed a good post-surgical prognostication and the prognostic nomogram proposed from our data might set the frame for future studies. We finally performed a specific cause of death analysis since patients both died of liver-related events and of non-liver-related events. By this approach, liver-related events were associated with HVPG and surgery type while extrahepatic deaths were related to ASA class. In this regard, ASA class behaves as a robust functional comorbid scale, better than other scales (i.e. Charlson) and overcomes relevant factors such as malignancy, at least for 1-year outcomes.

The observed mortality in our study cohort was $8 \%$ and $17 \%$ at 90 and 365 days, respectively, similar to other cohorts including elective surgeries ${ }^{8,15}$ but lower than most published series, which also include emergency procedures (13-27\% at 30 days and around $30 \%$ at 90 days)..$^{5,6,9,11}$ However, it must be noted that in elective procedures it is probably more relevant to balance the benefit/ratio risk before surgery if there are alternative strategies. Most of the surgical procedures were abdominal, with a small proportion of other surgical procedures. This has limited the possibility of a more precise risk assessment for each 
Table 3. Baseline variables associated with 1-year post-surgical mortality or transplantation.

\begin{tabular}{|c|c|c|c|c|}
\hline & Alive $(\mathbf{n}=116)$ & Death/OLT $(n=24)$ & Univariate $\boldsymbol{p}$ value & Multivariate $p$ value \\
\hline Age (years) & $62 \pm 10$ & $61 \pm 12$ & 0.636 & \\
\hline BMI $\left(\mathrm{kg} / \mathrm{m}^{2}\right)$ & $27.5 \pm 6.4$ & $25.3 \pm 4.7$ & 0.087 & \\
\hline ASA class II/III/IV (\%) & $35 / 61 / 4$ & $8 / 58 / 34$ & $<0.001$ & 0.008 \\
\hline Charlson comorbidity index (\%) & $5.0 \pm 1.8$ & $5.2 \pm 1.7$ & 0.673 & \\
\hline High-risk surgery (yes/no) (\%) & $29 / 71$ & $67 / 33$ & $<0.001$ & 0.006 \\
\hline Malignancy (yes/no) (\%) & $44 / 56$ & $50 / 50$ & 0.562 & \\
\hline Etiology alcohol/viral/others (\%) & $45 / 35 / 20$ & 29/58/13 & 0.135 & \\
\hline$\beta$-blockers (yes/no) (\%) & $38 / 62$ & $29 / 71$ & 0.398 & \\
\hline RAP (mmHg) & $6.3 \pm 3.7$ & $5.4 \pm 3.0$ & 0.220 & \\
\hline PAP $(\mathrm{mmHg})$ & $17.9 \pm 7.1$ & $16.8 \pm 5.7$ & 0.520 & \\
\hline PCP (mmHg) & $10.9 \pm 5.6$ & $9.6 \pm 6.0$ & 0.320 & \\
\hline Cardiac index $\left(\mathrm{L} / \mathrm{min} / \mathrm{m}^{2}\right)$ & $3.5 \pm 0.9$ & $3.6 \pm 0.7$ & 0.769 & \\
\hline MAP (mmHg) & $91 \pm 11$ & $87 \pm 14$ & 0.234 & \\
\hline SVRI $\left(\mathrm{dy} \cdot \mathrm{sec} / \mathrm{cm}^{5} \cdot \mathrm{m}^{2}\right)$ & $2064 \pm 635$ & $1850 \pm 444$ & 0.148 & \\
\hline PVRI, (dy $\left.\cdot \mathrm{sec} / \mathrm{cm}^{5} \cdot \mathrm{m}^{2}\right)$ & $172 \pm 91$ & $165 \pm 69$ & 0.759 & \\
\hline HVPG (mmHg) & $14.3 \pm 5.1$ & $18.4 \pm 5.3$ & 0.001 & 0.003 \\
\hline HVPG $\geq 10$ mmHg (yes/no) & $94 / 22$ & $22 / 2$ & 0.209 & \\
\hline HVPG >16 mmHg (yes/no) & $49 / 67$ & $16 / 8$ & 0.006 & 0.0016 \\
\hline HVPG $\geq 20$ mmHg (yes/no) & $18 / 98$ & $14 / 10$ & $<0.001$ & $<0.001$ \\
\hline Hepatic blood flow $(\mathrm{ml} / \mathrm{min})^{*}$ & $997 \pm 480$ & $799 \pm 370$ & 0.107 & \\
\hline IG fractional clearance* & $0.47 \pm 0.2$ & $0.45 \pm 0.1$ & 0.626 & \\
\hline $\mathrm{IGCl}(\mathrm{ml} / \mathrm{min})^{*}$ & $278 \pm 145$ & $209 \pm 85$ & 0.064 & \\
\hline Intrinsic IGCl (ml/min)* & $616 \pm 399$ & $390 \pm 175$ & 0.031 & 0.347 \\
\hline Plasma renin activity (ng/ml/h) & $1.0 \pm 1.5$ & $1.9 \pm 2.1$ & 0.040 & 0.239 \\
\hline Creatinine $(\mathrm{mg} / \mathrm{dl})$ & $1.0 \pm 0.3$ & $1.0 \pm 0.3$ & 0.573 & \\
\hline $\mathrm{Na}(\mathrm{mEq} / \mathrm{L})$ & $138 \pm 4$ & $139 \pm 4$ & 0.484 & \\
\hline $\operatorname{ALT}(\mathrm{U} / \mathrm{L})$ & $53 \pm 62$ & $42 \pm 27$ & 0.363 & \\
\hline Bilirubin (mg/dl) & $1.6 \pm 1.6$ & $2.1 \pm 1.7$ & 0.166 & \\
\hline Albumin $(\mathrm{g} / \mathrm{L})$ & $36.9 \pm 6.5$ & $33.7 \pm 5.5$ & 0.035 & 0.727 \\
\hline Hemoglobin $(\mathrm{g} / \mathrm{L})$ & $120 \pm 28$ & $108 \pm 24$ & 0.079 & \\
\hline Leucocyte $\left(\times 10^{9} / \mathrm{ml}\right)$ & $5.4 \pm 2.4$ & $5.4 \pm 2.4$ & 0.917 & \\
\hline Platelet $\left(\times 10^{12} / \mathrm{ml}\right)$ & $120 \pm 69$ & $116 \pm 60$ & 0.820 & \\
\hline INR & $1.2 \pm 0.2$ & $1.3 \pm 0.1$ & 0.438 & \\
\hline MELD score (UNOS) & $10.9 \pm 3.3$ & $11.8 \pm 2.9$ & 0.208 & \\
\hline MELD-Na score & $12.7 \pm 4.2$ & $13.3 \pm 3.8$ & 0.494 & \\
\hline Child-Pugh score & $6.3 \pm 1.5$ & $7.3 \pm 1.5$ & 0.006 & 0.775 \\
\hline Prev. decompensation (yes/no) (\%) & $55 / 45$ & $83 / 17$ & 0.019 & 0.178 \\
\hline
\end{tabular}

Results presented as mean \pm SD unless otherwise indicated. ${ }^{*} \mathrm{n}=115$ patients.

ALT, alanine aminotransferase; ASA, American Society of Anesthesiologists; HVPG, hepatic venous pressure gradient; IGCl, indocyanine green clearance; INR, international normalized ratio; MAP, mean arterial pressure; MELD, model for end-stage liver disease; OLT, orthotopic liver transplant; PAP, pulmonary artery pressure; PCP, pulmonary capillary pressure; RAP, right atrial pressure; SVRI, systemic vascular resistance index; PVRI, pulmonary vascular resistance index; UNOS, United Network for Organ Sharing.

Table 4. Prognostic accuracy for variables associated with 1-year post-surgical mortality in overall cohort and abdominal surgery subgroup. Models are both presented with HVPG as a continuous variable and as a dichotomous variable (HVPG $<$ or $\geq 20 \mathrm{mmHg}$ ).

\begin{tabular}{|c|c|c|c|c|}
\hline & \multicolumn{2}{|c|}{ Overall cohort $(\mathrm{N}=140)$} & \multicolumn{2}{|c|}{ Abdominal surgery $(n=121)$} \\
\hline & HR $(95 \% \mathrm{CI})$ & Performance & HR $(95 \% \mathrm{CI})$ & Performance \\
\hline \multicolumn{5}{|c|}{ Quantitative HVPG } \\
\hline ASA class & $\begin{array}{l}\text { III: } 2.98(0.7-13.2) \\
\text { IV: } 9.97(2.0-50.4)\end{array}$ & $\begin{array}{r}\text { C-statistic: } 90 \mathrm{~d}, 0.874 \\
180 \mathrm{~d}, 0.897 ; 365 \mathrm{~d}, 0.854\end{array}$ & $\begin{array}{l}\text { III: } 2.44(0.5-11.0) \\
\text { IV: } 16.39(2.8-95.3)\end{array}$ & $\begin{array}{r}\text { C-statistic: } 90 \mathrm{~d}, 0.909 ; \\
180 \mathrm{~d}, 0.895 ; 365 \mathrm{~d}, 0.840\end{array}$ \\
\hline High-risk surgery & $3.65(1.4-9.3)$ & AIC: 200.1 & $4.30(1.6-11.7)$ & AIC: 153.1 \\
\hline HVPG & $1.14(1.05-1.25)$ & & $1.18(1.07-1.30)$ & \\
\hline \multicolumn{5}{|c|}{ Dichotomous HVPG } \\
\hline ASA class & $\begin{array}{l}\text { III: } 3.78(0.9-16.8) \\
\text { IV: } 12.15(2.4-61.4)\end{array}$ & $\begin{array}{r}\text { C-statistic: } 90 \mathrm{~d}, 0.890 \\
180 \mathrm{~d}, 0.886 ; 365 \mathrm{~d}: 0.857\end{array}$ & $\begin{array}{l}\text { III: } 3.17(0.7-14.5) \\
\text { IV: } 16.84(2.8-100)\end{array}$ & $\begin{array}{r}\text { C-statistic: } 90 \text { d: } 0.909 ; \\
180 \text { d, } 0.890 ; 365 \text { d, } 0.840\end{array}$ \\
\hline High-risk surgery & $3.57(1.4-9.0)$ & AIC: 193.3 & $3.43(1.3-8.8)$ & AIC: 150.8 \\
\hline HPVG $\geq 20$ mmHg & $5.67(2.4-13.2)$ & & $6.37(2.49-16.31)$ & \\
\hline
\end{tabular}

AIC, Akaike information criterion; ASA, American Society of Anesthesiologists; HR, hazard ratio; HVPG, hepatic venous pressure gradient.

specific subtype of surgery. Although data on elective surgery is scarce, several studies showed that in an emergent basis, cardiovascular surgery is associated with the highest mortality risk, while orthopedic surgery may have an intermediate risk. $^{24,27-35}$ For this reason we decided to create a dichotomous surgical category (high risk/low risk) based on studies where orthopedic, laparoscopic and abdominal wall surgeries were associated with a lower post-surgical mortality compared to open abdominal or cardiovascular surgeries. ${ }^{10,11,23}$ It allowed to weigh the type of surgery in the model without the need 


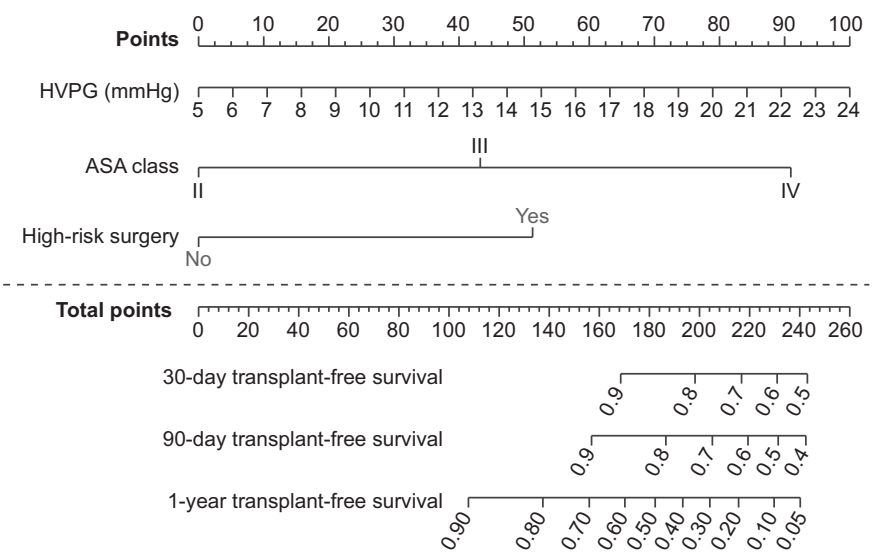

Fig. 2. Nomogram for 30-day, 90-day and 1-year post-surgical survival predictions according to the prognostic variables ASA class, low/high-risk surgery and HVPG. ASA, American Society of Anesthesiologists; HVPG, hepatic venous pressure gradient.

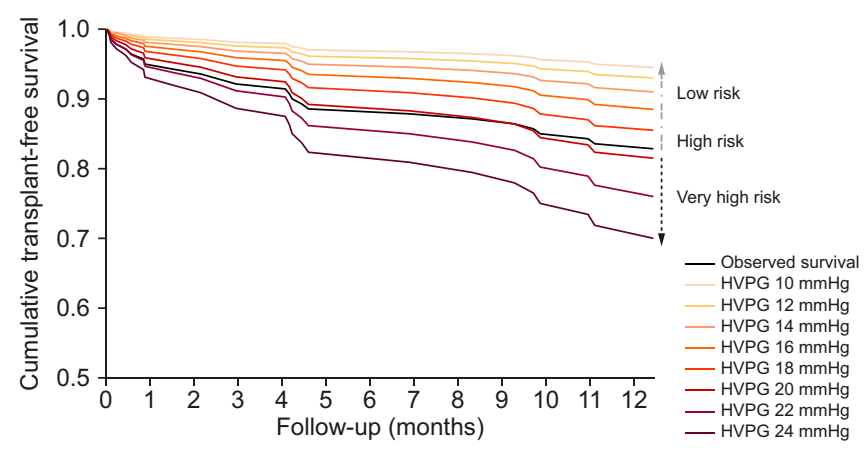

Fig. 3. Observed survival and modifying effects of HVPG adjusted by ASA class and type of surgery. HVPG values $>16 \mathrm{mmHg}$ associated a significantly higher risk of death and values $\geq 20 \mathrm{mmHg}$ identified the most at-risk patients. ASA, American Society of Anesthesiologists; HVPG, hepatic venous pressure gradient.

for a multi-categorical variable, with very small group sizes, which would have resulted in a loss of statistical power. Further supporting the rationale for such dichotomous categorization, higher surgical times (221 vs. $107 \mathrm{~min}$ ) and hospital length of stay (10.5 vs. 5 days) were observed in the high-risk surgical groups. To reinforce our findings, we also found the same independent prognostic factors when evaluating the more homogeneous subgroup of patients undergoing abdominal surgery.

A prominent and curious finding of our study is that MELD and Child-Pugh scores, the most used and validated scores in cirrhosis, ${ }^{15,36,37}$ including the surgical scenario, were not independent predictors of post-surgical mortality. This is especially intriguing for the MELD score, since it has been a prognostic factor in many surgical studies, one of which provided the foundation for one of the most widely used prognostic scores (Mayo Clinic model). ${ }^{5,11,15}$ Etiology, also included in this score, was not associated with prognosis nor other prognostic variables. A potential role for etiology could be better defined in larger studies. Regarding MELD, several factors may account for our negative results. First, our study only includes elective surgery and patients undergoing elective procedures are in more stable conditions and have better liver score values than those needing emergency surgery. Indeed, only $5 \%$ of the patients from our cohort had Child-Pugh class $\mathrm{C}$ cirrhosis, and the mean MELD

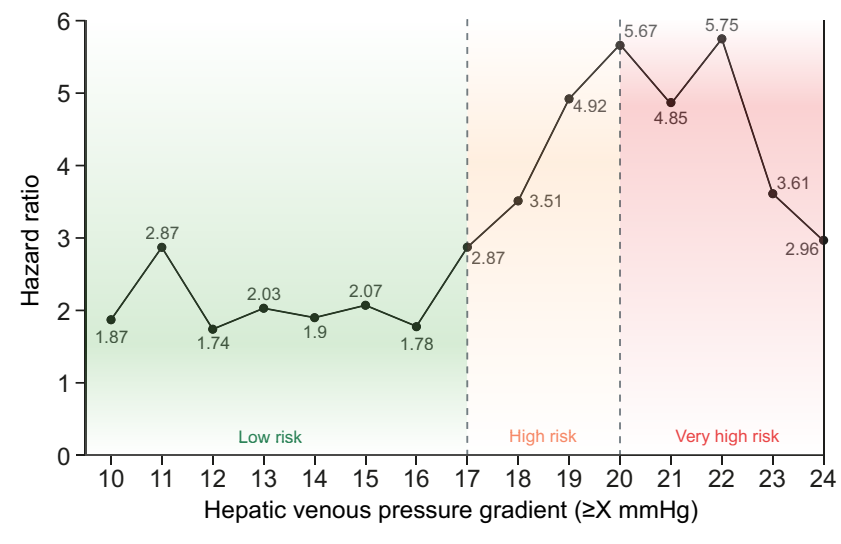

$\begin{array}{llllllllllllllll}\text { Events } & 22 & 22 & 20 & 20 & 19 & 18 & 16 & 16 & 15 & 14 & 14 & 11 & 6 & 4 & 3\end{array}$

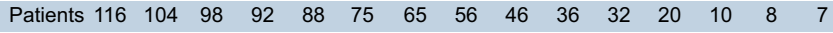

Fig. 4. Effect (hazard ratios) of different HVPG cut-offs on post-surgical 1year mortality/OLT adjusted by ASA class and type of surgery. HVPG values $>16 \mathrm{mmHg}$ were independent prognostic factors and values $\geq 20 \mathrm{mmHg}$ identified very high-risk patients (14 events in 32 individuals). ASA, American Society of Anesthesiologists; HVPG, hepatic venous pressure gradient; OLT, orthotopic liver transplant.

was 11. Therefore, our cohort does not include patients with high MELD or Child-Pugh scores, a fact that would explain the lower mortality in relation to previous studies including emergency surgery with worse MELD and Child scores. In fact, in the study by Teh et al., median MELD (not the United Network for Organ Sharing model) was clearly higher in emergency than in elective surgery: 12.2 vs. $7.9 .{ }^{11}$ Whether HVPG could also be an independent prognostic factor in emergency surgery remains open. Nevertheless, while HVPG can be easily measured prior to elective surgeries, it would be much more difficult to measure in the emergency setting.

Despite the initiation of the study many years ago, the participating hospitals are referral centers with totally contemporary surgical procedures. In this sense, a laparoscopic approach was applied whenever possible with a higher proportion of laparoscopic than open abdominal procedures (54 laparoscopies vs. 41 open). Laparoscopic surgeries included procedures requiring high expertise and outcomes in this subgroup were satisfactory with no events at 3 months and 3 events at 1 year. These findings are in accordance with previous studies reporting lower risks of decompensation and death with a laparoscopic approach. ${ }^{38-43}$ However, a proper comparison of laparoscopy vs. open abdominal surgeries cannot be done in our cohort since laparoscopy was generally the preferred approach and open procedures were used, including complex surgeries (urologic/ digestive), when laparoscopy was not feasible. Thus, despite our data not being conclusive, they support the laparoscopic approach for major abdominal surgery in cirrhosis whenever feasible, ideally in experienced centers.

In addition to mortality, we planned the analysis of 3-month persistent/worsening or de novo decompensation as a surrogate marker of increased risk of death during follow-up, as previously demonstrated in surgery for hepatocellular carcinoma. ${ }^{19}$ By selecting the 3-month period in assessing decompensation, we avoided the inclusion of patients with temporal ascites through drainages in abdominal surgery, which has an unknown clinical meaning. Factors independently associated with 3-month decompensation were high-risk surgery, Child-Pugh score and a lower BMI, reflecting the role of poor 
nutritional status on post-surgical outcomes. If entering MELD or MELD-Na instead of Child-Pugh as liver scores, variables associated with decompensation were BMI, type of surgery and HVPG. It must be pointed out that no patients with a Child-Pugh score of $5(\mathrm{n}=45$, mean HVPG $12.5 \mathrm{mmHg}$ ) had 3 months persistent decompensation. Further reinforcing the relevance of preserved liver function as a protective factor against decompensation, we found that in patients with indocyanine green clearance data (115 of 140 patients), an indocyanine green fractional clearance $>0.63$ protected patients from decompensation. Regarding HVPG, all patients presenting with decompensation had HVPG $\geq 10 \mathrm{mmHg}$. Anyhow, in the current study we were unable to demonstrate an association between 3-month persistent decompensation and future mortality. The low number of deaths within a short follow-up period and the strength of other prognostic variables (high-risk surgery, ASA and HVPG), which in part correlate with decompensation, may have diluted the impact of persistent decompensation on subsequent mortality.

Overall, the reported prognostic role of HVPG might be an argument for pre-surgical intervention (i.e. TIPS placement). Many uncontrolled retrospective studies have claimed that TIPS has a role in pre-surgical conditioning in cirrhotic patients. ${ }^{44-48}$ It has been postulated to diminish morbidity and mortality by decompressing the portal venous system. However, results are inconsistent across studies and despite the evidence that PHT plays a major role on post-surgical outcomes, potential risk thresholds to define patients in whom this strategy might be useful have not been defined. The present study provides concrete values from which studies on pre-surgical optimization could be planned: HVPG values above $16 \mathrm{mmHg}$ were independent prognostic factors and values $\geq 20 \mathrm{mmHg}$ identified an especially high-risk population. Our findings and proposed prognostic models (nomogram in Fig. 2) might even allow for a re-analysis of previous series, to confirm outcomes in patients with "risky" pre-surgical HVPG values and in whom TIPS were placed before surgery. In this regard, we included 3 patients with portosystemic shunts, since we aimed to assess the whole spectrum of PHT in our cohort. This low number of patients did not allow us to perform a sub-analysis, and even if these patients were excluded results remained unaltered. Therefore, the protective role of a pre-surgical TIPS remains open.

The present study has several limitations to point out. First, although a sample of 140 patients is large for a prospective cohort, external validation is desirable to strengthen our findings. In fact, the planned sample target was to include 200 patients in a period of 3 years, but a lower rate of inclusion than expected led us to finish the study with 140 patients. Prospective studies targeting HVPG "risky" populations (as defined by HVPG $>16 \mathrm{mmHg}$ ) should be designed to confirm our findings and to assess the potential benefit of lowering PHT. Second, despite inclusion of several types of surgery, most of them were abdominal. Based on sample size and previous literature, the classification of surgeries was simplified to low vs. high risk, though a more detailed categorization (and refined analysis) would be desirable in larger series. Third, the study period was dilated up to 10 years because of a low inclusion rate. However, looking at the type of procedures performed (high proportion of laparoscopy, for example), our cohort looks fully representative of contemporary surgical cohorts. Finally, our study only includes cirrhotic patients in whom elective surgery was considered by the medical-surgical team. Therefore, our results reflect a relatively stable spectrum of patients (predominant Child-Pugh class A and B) and our findings must be interpreted in this setting.

In conclusion, the present study provides evidence for the prognostic role of HVPG for post-surgical mortality in cirrhosis. HVPG values above $16 \mathrm{mmHg}$ were independent prognostic factors for mortality. These findings provide the framework for future studies assessing the potential role of pre-surgical TIPS in highly selected patients.

\section{Financial support}

Supported in part by grants from Ministerio de Educacion y Ciencia (SAF-2016-75767-R), and from Instituto de Salud Carlos III (PIE 15/00027). CIBERehd is funded by Instituto de Salud Carlos III. ER was recipient of a Río Hortega award (2012-2014), Instituto de Salud Carlos III.

\section{Conflict of interest}

The authors declare no conflicts of interest that pertain to this work.

Please refer to the accompanying ICMJE disclosure forms for further details.

\section{Authors' contributions}

Study concept and design: Joan Carles García-Pagán and Jaume Bosch. Screening, inclusion and follow-up of patients, performance of studies and acquisition of data: Isabel Cirera, Agustín Albillos, Wilma Debernardi-Venon, Juan G. Abraldes, Elba Llop, Alexandra Flores, Enric Reverter, Graciela Martínez-Palli, Annabel Blasi, Javier Martínez, Fanny Turon, Juan Carlos GarcíaValdecasas, Annalisa Berzigotti, Antoni M. de Lacy, Josep Fuster and Virginia Hernández-Gea. Analysis and interpretation of data: Enric Reverter, Joan Carles García-Pagán, Juan G. Abraldes and Jaume Bosch. Drafting of the manuscript: Enric Reverter and Joan Carles García-Pagán. Critical revision of the manuscript for important intellectual content: Jaume Bosch, Agustín Albillos, Juan G. Abraldes, Annalisa Berzigotti, Graciela Martínez-Palli, Annabel Blasi, Wilma Debernardi-Venon, Juan Carlos GarcíaValdecasas, Antoni M. de Lacy and Josep Fuster. Obtained funding: Joan Carles García-Pagán and Jaume Bosch. Guarantor of the article: Joan Carles García-Pagán. All the authors read and approved the final version of the paper.

\section{Supplementary data}

Supplementary data to this article can be found online at https://doi.org/10.1016/j.jhep.2019.07.007.

\section{References}

[1] Sheen IS, Liaw YF. The prevalence and incidence of cholecystolithiasis in patients with chronic liver diseases: a prospective study. Hepatology 1989;9:538-540.

[2] Fornari F, Imberti D, Squillante MM, Squassante L, Civardi G, Buscarini E, et al. Incidence of gallstones in a population of patients with cirrhosis. J Hepatol 1994;20:797-801.

[3] Conte D, Fraquelli M, Fornari F, Lodi L, Bodini P, Buscarini L. Close relation between cirrhosis and gallstones: cross-sectional and longitudinal survey. Arch Intern Med 1999;159:49-52.

[4] Belghiti J, Durand F. Abdominal wall hernias in the setting of cirrhosis. Semin Liver Dis 1997;17:219-226. 
[5] Northup PG, Wanamaker RC, Lee VD, Adams RB, Berg CL. Model for endstage liver disease (MELD) predicts nontransplant surgical mortality in patients with cirrhosis. Ann Surg 2005;242:244-251.

[6] Ziser A, Plevak DJ, Wiesner RH, Rakela J, Offord KP, Brown DL. Morbidity and mortality in cirrhotic patients undergoing anesthesia and surgery. Anesthesiology 1999;90:42-53.

[7] Artinyan A, Marshall CL, Balentine CJ, Albo D, Orcutt ST, Awad SS, et al. Clinical outcomes of oncologic gastrointestinal resections in patients with cirrhosis. Cancer 2012;118:3494-3500.

[8] Telem DA, Schiano T, Goldstone R, Han DK, Buch KE, Chin EH, et al. Factors that predict outcome of abdominal operations in patients with advanced cirrhosis. Clin Gastroenterol Hepatol 2010;8:451-457.

[9] Nguyen GC, Correia AJ, Thuluvath PJ. The impact of cirrhosis and portal hypertension on mortality following colorectal surgery: a nationwide, population-based study. Dis Colon Rectum 2009;52:1367-1374.

[10] Csikesz NG, Nguyen LN, Tseng JF, Shah SA. Nationwide volume and mortality after elective surgery in cirrhotic patients. J Am Coll Surg 2009;208:96-103.

[11] Teh SH, Nagorney DM, Stevens SR, Offord KP, Therneau TM, Plevak DJ, et al. Risk factors for mortality after surgery in patients with cirrhosis. Gastroenterology 2007;132:1261-1269.

[12] de Goede B, Klitsie PJ, Lange JF, Metselaar HJ, Kazemier G. Morbidity and mortality related to non-hepatic surgery in patients with liver cirrhosis: a systematic review. Best Pract Res Clin Gastroenterol 2012;26: 47-59.

[13] Costa BP, Castro Sousa F, Serôdio M, Carvalho C. Value of MELD and MELD-based indices in surgical risk evaluation of cirrhotic patients: retrospective analysis of 190 cases. World J Surg 2009;33:1711-1719.

[14] Mansour A, Watson W, Shayani V, Pickleman J. Abdominal operations in patients with cirrhosis: still a major surgical challenge. Surgery 1997; 122:730-5-6.

[15] Kim SY, Yim HJ, Park SM, Kim JH, Jung SW, Seo YS, et al. Validation of a Mayo post-operative mortality risk prediction model in Korean cirrhotic patients. Liver Int 2011;31:222-228.

[16] Bosch J, García-Pagán JC. Complications of cirrhosis. I. Portal hypertension. J Hepatol 2000;32:141-156.

[17] Groszmann RJ, Garcia-Tsao G, Bosch J, Grace ND, Burroughs AK, Planas R, et al. Beta-blockers to prevent gastroesophageal varices in patient with cirrhosis. N Engl J Med 2005:353:2254-2261.

[18] Bosch J, Garcia-Pagán JC, Berzigotti A, Abraldes J. Measurement of portal pressure and its role in the management of chronic liver disease. Semin Liver Dis 2006:26:348-362.

[19] Bruix J, Castells A, Bosch J, Feu F, Fuster J, Visa J, et al. Surgical ressection of hepatocellular carcinoma in cirrhotic patients: prognostic value of preoperative portal pressure. Gastroenterology 1996:1018-1022.

[20] Lau H, Man K, Fan ST, Yu WC, Lo CM, Wong J. Evaluation of preoperative hepatic function in patients with hepatocellular carcinoma undergoing hepatectomy. Br J Surg 1997;84:1255-1259.

[21] Bosch J, Abraldes JG, Berzigotti A, García-Pagan JC. The clinical use of HVPG measurements in chronic liver disease. Nat Rev Gastroenterol Hepatol 2009:6:573-582.

[22] García-Pagán JC, Feu F, Luca A, Fernández M, Pizcueta P, Bosch J, et al. Nicardipine increases hepatic blood flow and the hepatic clearance of indocyanine green in patients with cirrhosis. J Hepatol 1994;20:792-796.

[23] Neeff H, Mariaskin D, Spangenberg H-C, Hopt UT, Makowiec F. Perioperative mortality after non-hepatic general surgery in patients with liver cirrhosis: an analysis of 138 operations in the 2000s using Child and MELD scores. J Gastrointest Surg 2011;15:1-11.

[24] Hsieh PH, Chen LH, Lee MS, Chen CH, Yang WESC. Hip arthroplasty in patients with cirrhosis of the liver. J Bone Jt Surg Br 2003;85:818-821.

[25] Meunier OK, Mucci MDS, Quentin MDV, Azoulay CR, Arnaud MDJP, Hamy MDA. Colorectal surgery in cirrhotic patients: assessment of operative morbidity and mortality. Dis Colon Rectum 2008;1231:1225-1231.

[26] Berzigotti A, Seijo S, Arena U, Abraldes JG, Vizzutti F, García-Pagán JC, et al. Elastography, spleen size, and platelet count identify portal hypertension in patients with compensated cirrhosis. Gastroenterology 2013:144:102-111.

[27] Cohen SM, Te HS, Levitsky J. Operative risk of total hip and knee arthroplasty in cirrhotic patients. J Arthroplasty 2005;20:460-466.
[28] Morisaki A, Hosono M, Sasaki Y, Kubo S, Hirai H, Suehiro S, et al. Risk factor analysis in patients with liver cirrhosis undergoing cardiovascular operations. Ann Thorac Surg 2010;89:811-817.

[29] Modi A, Vohra HA, Barlow CW. Do patients with liver cirrhosis undergoing cardiac surgery have acceptable outcomes?. Interact Cardiovasc Thorac Surg 2010;11:630-634.

[30] Suman A, Barnes DS, Zein NN, Levinthal GN, Connor JT, Carey WD. Predicting outcome after cardiac surgery in patients with cirrhosis: a comparison of Child-Pugh and MELD scores. Clin Gastroenterol Hepatol 2004;2:719-723.

[31] Macaron C, Hanouneh IA, Suman A, Lopez R, Johnston D, Carey WW. Safety of cardiac surgery for patients with cirrhosis and Child-Pugh scores less than 8. Clin Gastroenterol Hepatol 2012;10:535-539.

[32] Hayashida N, Shoujima T, Teshima H, Yokokura Y, Takagi K, Tomoeda H, et al. Clinical outcome after cardiac operations in patients with cirrhosis. Ann Thorac Surg 2004;77:500-505.

[33] Thielmann M, Mechmet A, Neuhäuser M, Wendt D, Tossios P, Canbay A, et al. Risk prediction and outcomes in patients with liver cirrhosis undergoing open-heart surgery. Eur J Cardiothorac Surg 2010;38: 592-599.

[34] Filsoufi F, Salzberg SP, Rahmanian PB, Schiano TD, Elsiesy H, Squire A, et al. Early and late outcome of cardiac surgery in patients with liver cirrhosis. Liver Transpl 2007:990-995.

[35] Shih L-Y, Cheng C-Y, Chang C-H, Hsu K-Y, Hsu RW-W, Shih H-N. Total knee arthroplasty in patients with liver cirrhosis. J Bone Joint Surg Am 2004;86-A:335-341.

[36] Hoteit M-A, Ghazale A-H, Bain A-J, Rosenberg E-S, Easley K-A, Anania FA, et al. Model for end-stage liver disease score versus Child score in predicting the outcome of surgical procedures in patients with cirrhosis. World J Gastroenterol 2008;14:1774-1780.

[37] Cho HC, Jung HY, Sinn DH, Choi MS, Koh KC, Paik SW, et al. Mortality after surgery in patients with liver cirrhosis: comparison of ChildTurcotte-Pugh, MELD and MELDNa score. Eur J Gastroenterol Hepatol 2011;23:51-59.

[38] Martınez JL, Rivas H, Delgado S, Castells A, Pique JM, Lacy AM. Laparoscopic-assisted colectomy in patients with liver cirrhosis. Surg Endosc 2004:1071-1074.

[39] Shaikh AR, Muneer A. Laparoscopic cholecystectomy in cirrhotic patients. JSLS 2009;13:592-596.

[40] Pavlidis TE, Symeonidis NG, Psarras K, Skouras C, Kontoulis TM, Ballas K, et al. Laparoscopic cholecystectomy in patients with cirrhosis of the liver and symptomatic cholelithiasis. JSLS 2009:13:342-345.

[41] Yeh CN, Chen MF, Jan YY. Laparoscopic cholecystectomy in 226 cirrhotic patients. Experience of a single center in Taiwan. Surg Endosc 2002;16:1583-1587.

[42] Currò G, Iapichino G, Melita G, Lorenzini C, Cucinotta E. Laparoscopic cholecystectomy in Child-Pugh class C cirrhotic patients. JSLS 2005;9:311-315.

[43] Poggio JL, Rowland CM, Gores GJ, Nagorney DM, Donohue JH. A comparison of laparoscopic and open cholecystectomy in patients with compensated cirrhosis and symptomatic gallstone disease. Surgery 2000; $127: 405-411$.

[44] Fares N, Robic M-A, Péron J-M, Muscari F, Otal P, Suc B, et al. Transjugular intrahepatic portosystemic shunt placement before abdominal intervention in cirrhotic patients with portal hypertension. Eur J Gastroenterol Hepatol 2018:30:21-26.

[45] Azoulay D, Buabse F, Damiano I, Smail A, Ichai P, Dannaoui M, et al. Neoadjuvant transjugular intrahepatic portosystemic shunt: a solution for extrahepatic abdominal operation in cirrhotic patients with severe portal hypertension. J Am Coll Surg 2001;193:46-51.

[46] Schlenker C, Johnson S, Trotter JF. Preoperative transjugular intrahepatic portosystemic shunt (TIPS) for cirrhotic patients undergoing abdominal and pelvic surgeries. Surg Endosc 2009;23:1594-1598.

[47] Menahem B, Lubrano J, Desjouis A, Lepennec V, Lebreton G, Alves A. Transjugular intrahepatic portosystemic shunt placement increases feasibility of colorectal surgery in cirrhotic patients with severe portal hypertension. Dig Liver Dis 2015;47:81-84.

[48] Kim JJ, Dasika NL, Yu E, Fontana RJ. Cirrhotic patients with a transjugular intrahepatic portosystemic shunt undergoing major extrahepatic surgery. J Clin Gastroenterol 2009;43:574-579. 